\title{
Hydrodynamic Analysis of Fluid Obstruction Around Different Geometric Bodies
}

\author{
E. Žic( ${ }^{(1)}$, P. Černeka(2), I. Biluš(3) \\ (1) Faculty of Civil Engineering, Division of Hydromechanics, University of Rijeka, Radmile Matejčić 3, Rijeka, 51000, CROATIA \\ e-mail: elvis.zic@uniri.hr \\ (2) Faculty of Civil Engineering, University of Rijeka, Radmile Matejčić 3, Rijeka, 51000, CROATIA \\ (3) Faculty of Mechanical Engineering, University of Maribor, Smetanova ulica 17, Maribor, SI-2000, SLOVENIA
}

\section{SUMMARY}

The aim of this paper is to conduct a hydrodynamic analysis of fluid flow around different geometric bodies on the laboratory physical model HM 133 of the Gunt Company from Hamburg and to show the formation of boundary layer and separation points on the observed bodies. The paper covers the field of real fluid dynamics which includes a description of laminar and turbulent flow together with a Reynolds number. A detailed representation of the boundary layer, its characteristics, and its structure are included. The body models in the paper used on the HM 133 physical model were an oblong straight body and cylindrical bodies with $6 \mathrm{~mm}, 12 \mathrm{~mm}, 18$ $\mathrm{mm}$, and $24 \mathrm{~mm}$ in diameter. The research was conducted in the Hydrotechnical Laboratory of the Faculty of Civil Engineering, University of Rijeka. Hydrodynamic analyses were made based on the tested body models on the physical model HM 133 together with the analyses on the numerical models performed using the ANSYS Fluent software.

KEY WORDS: $\quad$ hydrodynamic analysis; physical model HM133; boundary layer; ANSYS Fluent.

\section{INTRODUCTION}

Today, more and more attention is being paid to hydrodynamic analyses within the field of Computational Fluid Dynamics (CFD) since they are an important part of industrial technology and development. The fluid flow around cylindrical objects has been the topic of many experimental and numerical studies because of its importance in engineering research. Circular, square, and rectangular columns or pillars are widely used in mechanical engineering, civil engineering, and shipbuilding through structures such as coastal platforms, transmission lines, bridge piers, and similar (Žic, 2019) [1]. Fluid obstruction around individual objects shows some important physical phenomena such as vortex formation and turbulence. Several practical mechanical properties, including drag force and buoyancy force, as well as pressure coefficients significantly influence on the vortex formation mechanism (Bimbato et al., 2011) [2]. To understand more clearly the complexity of laminar and turbulent flows around 
geometric bodies innovative numerical techniques and methods have been introduced. CFD is a useful tool for demonstrating the flow field based on which vector and contour representations of changes in physical quantities can be displayed, as well as their numerical simulations in space and time. Understanding the phenomenon of vortex release in various forms is extremely important for its physical application and can cause serious harm (Ausoni, 2009) [3]. Although the vortex scale is known, the nature of the vortex process is still not fully recognized. Błazik-Borow et al. (2011) [4] state that many methods have been proposed over the years to control the dynamics of eddy currents, but unfortunately turbulence models have not yet properly described the phenomenon of turbulent "separation" of eddies. Leonardo da Vinci was one of the first to describe the vortex separation phenomenon by drawing several fairly accurate sketches of the vortex formation in the flow behind an arbitrary body. The creation of a vortex around the body was described by Theodore von Karman in 1911, defining it as periodic separable pairs of alternating vortices on a body around which fluid is obstructed, generating an oscillating flow that occurs when fluid flows around a body with a certain velocity, depending on the size and shape of the body (Ausoni, 2009) [3]. The eddy separation is determined by the viscosity of the fluid passing over the body and the Reynolds number for the same fluid. In the area with a larger Reynolds number, the flow is dominated by inertial forces and turbulence occurs, while in the area with a low Reynolds number, the viscous force is dominant and laminar flow develops.

\section{PREVIOUS RESEARCH}

Numerous experimental and computational studies in the literature have been conducted on the fluid flow around production bodies of various shapes to explicitly demonstrate the formation of vortices at different values of Reynolds number (Re). Zdravkovich (2003) [5] defines a series of transitions from laminar to turbulent regime in various areas of flow fields around cylinders. Oudheusden et al. (2005) [6] performed an experimental study of vorticity near a square cylinder using PIV technology on experiments in which $R e$ was taken with values of $4^{*} 10^{3}, 1^{*} 10^{4}$, and $2^{*} 10^{4}$. Benim et al. (2007) [7] numerically investigated turbulent flow in circular cylinders using the SST $k$ - $\omega$ turbulent model with a wide range of Re numbers from $10^{4}$ to $5^{*} 10^{6}$. Rajani et al. (2009) [8] investigated the analysis of fluid obstruction at laminar flow around a circular cylinder for $R e$ in the range from 100 to 400. Ong et al. (2009) [9] performed fluid flow studies around smooth circular cylinders for $R e$ with values of $1^{*} 10^{6}, 2^{*} 10^{6}$, and $3.6^{*} 10^{6}$ using 2D Reynolds averaged Navier-Stokes (RANS) equations. They tested the applicability of a standard $k-\varepsilon$ turbulent model for engineering applications in the supercritical regime and upper transient flow regime. Gera et al. (2010) [10] researched 2D unsteady flows by the FEM method around a quadrilateral cylinder for $R e$ in the range of 50 to 250 . Bai and Li (2011) [11] simulated the hydrodynamic characteristics of circular cylinders in 2D unsteady flows using the CFD technique for $R e=200$. The very reliable values of the pressure distribution coefficients, the coefficients of friction and buoyancy, and the Strouhal number were obtained. Kozlov et al. (2011) [12] simulated 2D flow on circular cylindrical objects for Re ranging from 5 to 200 by applying fast Fourier transforms used to solve the Poisson equation for rectangular networks with complex geometries. Mustto and Bodstien (2011) [13] proposed a numerical model to predict the flow characteristics around a circular cylinder for Re between $10^{4}$ and $6^{*} 10^{5}$ using the Lagrangian vortex technique without network in combination with the LES model to simulate motion. Wornom et al. (2011) [14] conducted a computational analysis of the flow on a circular cylinder at a subcritical regime with $R e$ up to 
$2 * 10^{5}$ using a variational-multiscale LES method. In their work, Yuce and Kareem (2016) [15] conducted a hydrodynamic analysis of fluid obstruction around columns of circular and square transverse profile to protect coastal structures and bridges. The analysis was performed for the range of $R e=2$ (laminar flow) to strong turbulent flows $\left(R e=4^{*} 10^{6}\right.$ ). Numerical simulation analyses for 2D flow were performed using the SST $k$ - $\omega$ turbulence model in Ansys Fluent software.

\section{DESCRIPTION OF FLUID MOTION ON A FLAT PLATE}

The gradient of velocity field tensor defines how the vector field changes in a particular direction. The change in position is determined by the position of vector $\vec{r}$ and the angular velocity $\vec{\omega}$. A particle can undergo four different forms of deformation: translation, rotation, linear and angular deformation. All four types of deformation usually occur simultaneously which complicates the study of fluid dynamics (Žic, 2019) [1]. The displacement and rotation of a particle only change their position while their dimensions remain constant in space. The deformations at which the shape of the particle changes are linear and angular deformations. Linear deformation can cause volume changes in compressible fluids, whereby the volume of such a particle may increase or decrease depending on the increase or decrease in density. When fluid flows on a flat plate, at a short distance $x$ from the plate the fluid moves at a certain velocity. Due to the viscosity, a boundary layer is created near the plate in which the velocity changes continuously from the value $v=0 \mathrm{~m} / \mathrm{s}$ to some maximum value. With increasing $R e$ number the laminar boundary layer becomes a turbulent boundary layer. The range of $R e$ values at which the boundary layer changes is $3^{*} 10^{6}$ to $4^{*} 10^{6}$ (Bukurov and Žic, 2017) [16]. In the region of the turbulent boundary layer, a thin viscous sublayer is formed which exhibits high velocity gradients and internal friction. As the flow changes from laminar to turbulent, the thickness of the boundary layer increases downstream of the leading edge. The thickness of the boundary layer $\delta$ on the flat plate depends on the distance $x$ from the front edge of the flat plate and the flow regime. In the case of a smooth and stable flow occurring near the edge, the thickness of the boundary layer is determined by the expression:

$$
\delta(x)=\frac{c \cdot x}{\left(R e_{X}\right)^{1 / 2}}=\left(\frac{c \cdot x^{1 / 2}}{v_{\infty}^{1 / 2}}\right) \cdot\left(\frac{\mu}{\rho^{1 / 2}}\right)
$$

where $c$ is a constant, $\rho$ is the fluid density, $R e_{X}=\frac{\rho v_{\infty} X}{\mu}$ is Reynolds number, $v_{\infty}$ represents the free-flow velocity, while $\mu$ is a dynamic viscosity. If there is a low viscosity (or the $R e_{x}$ is higher) then the boundary layer growth is slower. The friction on the substrate depends on the coefficient of friction resistance $c_{f}$ which depends on the change in the thickness of the boundary layer along the substrate. The resistance of a particle depends on its geometric characteristics, the velocity at which the particle moves through the fluid, the wall roughness, the density and viscosity of the fluid. The boundary layer will affect the friction resistance and the coefficient of resistance depends on whether the substrate is rough or smooth. The formation of the boundary layer is also affected by the pressure gradient that changes the velocity profile. Observing the flow in the $x y$ plane during convergent flow, it is noticed that as the velocity increases in the $x$ direction, the pressure gradient becomes negative and the boundary layer is thin $(d U / d x>0, d p / d x<0)$. As the velocities increase, the pressure drops, whereby the turbulent vortices cannot remain in the boundary layer. The vortices further 
transmit longitudinal currents. In such a situation, the thickness of the boundary layer is relatively thin. Unlike acceleration, the boundary layer thickness increases during deceleration. With a slowing divergent flow, the pressure increases and becomes positive. The point at which the boundary layer separates from the flat plate is called the separation point. It is located in the area of decelerating flow $d p / d l>0$. At the separation point, the velocity gradient becomes zero $d v / d l=0$ (Bukurov and Žic, 2017) [16].

\section{LABORATORY PHYSICAL MODEL HM 133}

The experimental studies in this paper are conducted on the physical model HM 133 of Gunt Company from Hamburg (Figure 1). The model is used to visualize flow fields, respectively laminar and turbulent flow processes in a rectangular channel. In the working section of the physical model, various 3D geometric bodies are placed on which the fluid flow around the body, the formation of the boundary layer, and the appearance of the point of separation are analysed (Černeka, 2019) [17]. The physical model HM 133 is a device that uses electricity to drive the pump (water circulation) and the current controller (to generate bubbles). It consists of a $900^{*} 300^{*} 220 \mathrm{~mm}$ polystyrene container. It circulates fluid (Figure $2 \mathrm{a}$ ) from the upper inlet to the lower outlet tank. Glass balls are evenly spaced at the bottom of the inlet tank to stabilize the flow. The tank is filled with a maximum of 6 liters of water until the inlet and outlet tank are filled up to approximately $2 \mathrm{~cm}$ below the top edge of the tank. A small flow stabilizer plate is placed vertically so that a steady and uniform flow can develop (Figure 2b). The continuous circulation of water is ensured by the pump hidden in the housing below the tank and the flow rate is simply adjusted by regulating the speed of the pump via the regulator. The regulator can adjust the intensity of bubble production as well as the formation of pause intervals in the process of their generation (Černeka, 2019) [17].

In the paper, the constant bubble production, without pauses, is considered. The model operates on the basis of electrolysis. The two electrodes are immersed in water and powered by electricity. The stainless steel plate serves as the anode and the thin platinum wire acts as the cathode. If the cathode is closer to the anode, the more hydrogen bubbles are generated through the wire (Figure 2). Increasing the current on the regulator leads to an increase in the production of hydrogen bubbles. The area of the measuring section is illuminated by white LEDs on both sides of the channel. The measuring part has dimensions of $560^{*} 150^{*} 40 \mathrm{~mm}$. For better visualization of the bubbles Glauber's salt (sodium sulfate decahydrate, $\mathrm{Na}_{2} \mathrm{SO}_{4}$ * $10 \mathrm{H}_{2} \mathrm{O}$ ) is added to the water.
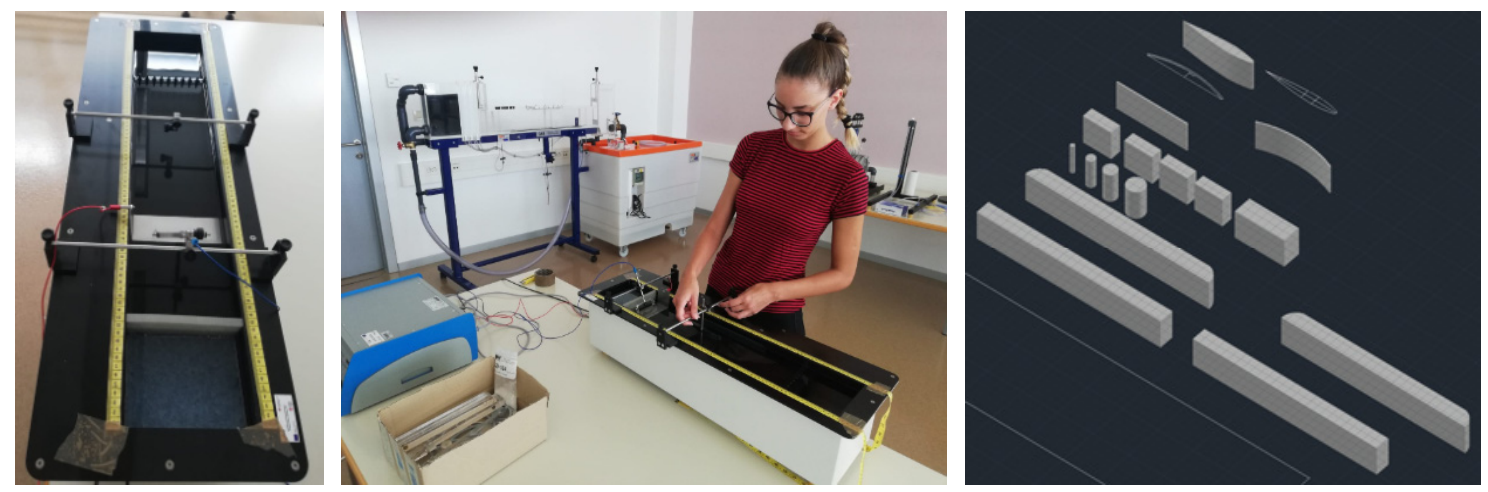
Fig. 1 Conducting experiments on the physical model HM 133 (left and center figures) and shapes of geometric bodies for testing on the physical model (right figure) [Foto: E. Žic]
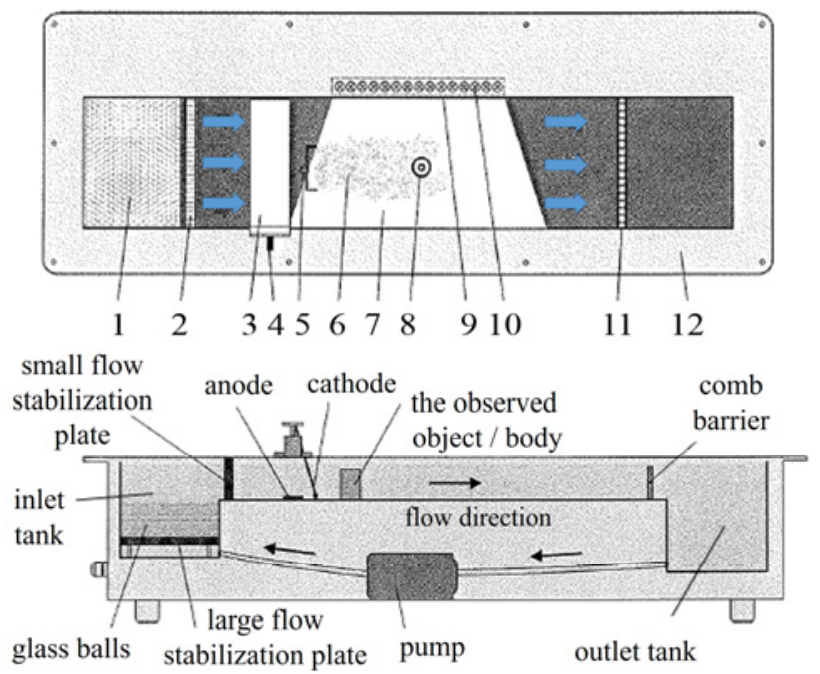

Fig. 2 Physical model HM 133; a) ground plan, b) side view (Pamić et al., 2018) [18]

On the plan view in Figure 2a) number 12 indicates the polystyrene container within which the flow takes place. Glass balls are correctly arranged in the inlet tank (mark 1). Mark 2 shows the flow stabilization panel. For the electrolysis process a stainless steel plate is used (marks 3 and 4) which is immersed in water, ie. the anode and the cathode (5). Electrolysis produces hydrogen bubbles (6) visible clearly in the bright light (7). The number 8 represents the geometric body that is placed inside the measuring section. Mark 9 shows windows and mark 10 LEDs. A comb barrier (11) is used to regulate the water level.

\subsection{TESTS CONDUCTED ON THE PHYSICAL MODEL HM 133}

The test is performed on two different geometric bodies: an elongated straight body (dimension $\left.L: w_{1}: H=260 * 20 * 40 \mathrm{~mm}\right)$ and four cylindrical bodies of different diameters $\left(d_{1}=6\right.$ $\mathrm{mm}, d_{2}=12 \mathrm{~mm}, d_{3}=18 \mathrm{~mm}, d_{4}=24 \mathrm{~mm}$ ). Each of these geometric bodies makes up one complete model, which is tested separately in such a way that the same geometric bodies are positioned in several different positions or at different angles to give a better view of the fluid flow around them. The input flow velocity, on the basis of which the hydrodynamic analysis is performed on physical models, is taken from the values of $0,25 \mathrm{~m} / \mathrm{s}$ (for the elongated body models) and $0,5 \mathrm{~m} / \mathrm{s}$ (for the models with a cylindric body). The physical model HM 133 enables flow velocity in the working section of up to $0,5 \mathrm{~m} / \mathrm{s}$ to be achieved via a speed controller. Velocity measurements at individual critical points within the working section of the physical model are performed using a classical Pitot tube (using Toriccelli's formula, $v=\sqrt{2 g h}$ ) in order to ultimately verify the values on numerical models for flow velocity around individual examined geometric bodies (Table 1, Figure 3c), Figure 4c), Figure 7c), Figure 10c)). Additional control of the calculation is performed using a small ball of styrofoam of negligible mass traveling down the water stream. The change in the styrofoam ball path per unit time is measured. 
Table 1 Verification of flow velocity on physical (measured) and numerical model (calculated)

\begin{tabular}{|c|c|c|c|}
\hline \multicolumn{4}{|c|}{ Model 1 - oblong straight body (angle of 20 $)$} \\
\hline $\begin{array}{c}\text { Measuring } \\
\text { point }\end{array}$ & $\begin{array}{c}\text { Height in Pitot } \\
\text { tube, } h[\mathrm{~mm}]\end{array}$ & $\begin{array}{c}\text { Physical model, } v \\
{[\mathrm{~m} / \mathrm{s}]}\end{array}$ & $\begin{array}{c}\text { Numerical model, } \\
\mathrm{[m} / \mathrm{s}]\end{array}$ \\
\hline 1 & 44,0 & 0,93 & 1,02 \\
\hline 2 & 8,0 & 0,40 & 0,42 \\
\hline 3 & 3,5 & 0,26 & 0,28 \\
\hline 4 & 9,0 & 0,42 & 0,37 \\
\hline \multicolumn{4}{|c|}{ Model 2 - cylindrical body $(\mathrm{d}=18 \mathrm{~mm}$ ) } \\
\hline $\begin{array}{c}\text { Measuring } \\
\text { point }\end{array}$ & $\begin{array}{c}\text { Height in Pitot } \\
\text { tube, } h[\mathrm{~mm}]\end{array}$ & $\begin{array}{c}\text { Physical model, } v \\
{[\mathrm{~m} / \mathrm{s}]}\end{array}$ & $\begin{array}{c}\text { Numerical model, } \\
\text { [m/s] }\end{array}$ \\
\hline 1 & 14 & 0,52 & 0,55 \\
\hline 2 & 16 & 0,56 & 0,58 \\
\hline 3 & 24 & 0,69 & 0,66 \\
\hline 4 & 19 & 0,61 & 0,63 \\
\hline
\end{tabular}

\subsubsection{MODEL 1: OBLONG STRAIGHT BODY}

The first submodel of the oblong flat body (submodel 1.1) is set in the flow direction $\left(0^{\circ}\right)$. When fluid comes to the model, there is a great deal of resistance which causes a lot of pressure and a decrease in velocity in the front of the model. The created boundary layer is clearly visible on the thus placed body (Figure 3a). The oblong straight body is positioned symmetrically with respect to the flow so that the separation point appears almost symmetrically. Immediately behind the tops of the body, there is a significant turbulence.
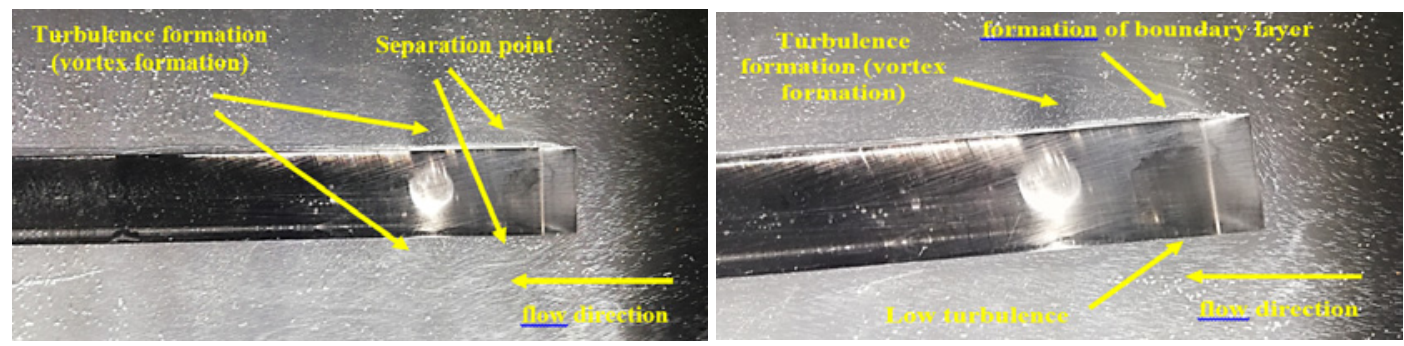

a)

b)
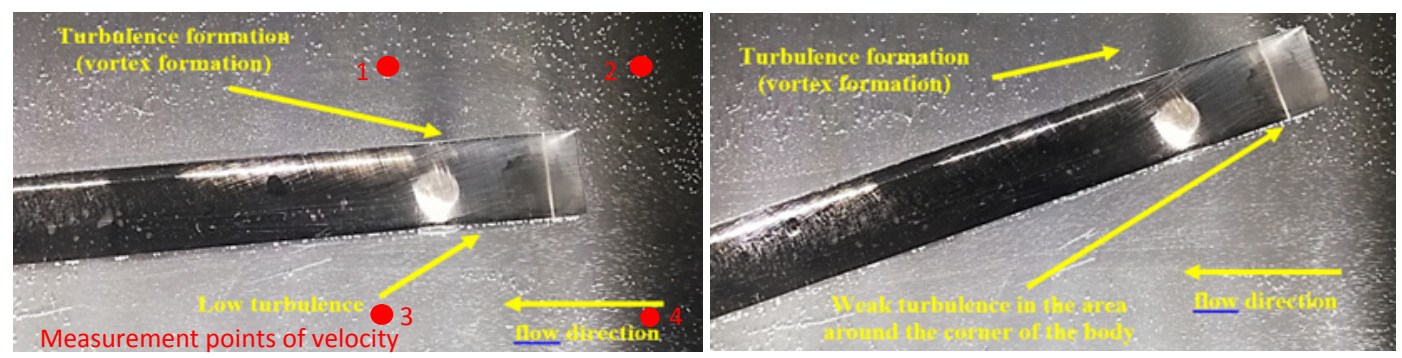

c)

d)

Fig. 3 Fluid flow around an oblong straight body in the direction of flow motion, a) submodel 1.1, b) submodel 1.2, c) submodel 1.3, d) submodel 1.4 [Foto: P. Černeka] 
The second submodel of the oblong straight body (submodel 1.2) is inclined at an angle of $10^{\circ}$ with respect to the flow direction. With the arrival of flow, the streamlines deform on the surface of the body causing turbulence on the tops of the body. As the body is no symmetrically positioned in the flow direction there is no symmetrical separation of the boundary layer. In Figure $3 \mathrm{~b}$ ) it can be seen that the boundary layer is thicker from the upper side relative to the lower one. Thus, a slight appearance of the top can be noticed, while the fluid flows almost laminarly from the underside of the walls on the oblong straight body. The third submodel of the oblong straight body (submodel 1.3) is set at an angle of $20^{\circ}$ with respect to the flow direction (Figure 3c). Due to the viscosity of the fluid, a boundary layer is formed along the wall of the body. When the streamlines come to the body their direction is disturbed and vortices occur. The vortices are more clearly visible on the upper side of the body than on the lower because on the upper side the fluid abruptly encounters an obstacle, i.e. the front surface of the body, which creates turbulence, while the fluid on the lower side slides down the wall of the body at an angle of $20^{\circ}$ so no significant formation of vortices is observed on the underside of the body walls. The last submodel of the oblong straight body (submodel 1.4) is inclined at an angle of $25^{\circ}$ with respect to the flow direction (Figure $3 \mathrm{~d}$ ). This submodel exhibits the highest turbulence formation, especially at the upper part near the top of the oblong straight body. The higher intensity vortices appear on the upper side causing deformation of the original streamlines, while on the underside no vortices occur due to a more favorable position of the body when fluid comes.

\subsubsection{MODEL 2: CYLINDRICAL BODIES WITH DIFFERENT DIAMETERS}

The first submodel (submodel 2.1) is a cylindrical body with a diameter of $6 \mathrm{~mm}$ (Figure 4a). The body is positioned in the middle of the measuring section in the direction of the fluid flow (at $v=0,5 \mathrm{~m} / \mathrm{s}$ ). Due to the small diameter, no significant turbulence, and no significant hydrodynamic resistance are observed. The fluid flow becomes deformed upon contact with the body, but due to the low velocity and the size of the body itself, the fluid flow quickly regains its original character (laminar flow).

The second submodel (submodel 2.2) is a cylindrical body of $12 \mathrm{~mm}$ diameter placed at the center of the fluid flow (Figure 4b). Significant pressures occur in front of the body, while slight vortices appear behind the body. The figure shows that a boundary layer is formed equally on both sides of the body. The third submodel of the cylindrical body (submodel 2.3) of $18 \mathrm{~mm}$ diameter is also placed at the center of the fluid flow (Figure 4c). If the diameter of the analysed body grows the vortices are getting bigger behind the body. Separation of the boundary layer takes place in an area longer than a half of the diameter of the cylindrical body. The last tested submodel is a cylindrical body of $24 \mathrm{~mm}$ in diameter (submodel 2.4) located in the middle of the flow motion (Figure 4d). The characteristics that can be observed are similar to those of the previous submodels 2.2. and 2.3. The cylindrical bodies with a larger diameter are better for the observation of the vortex formed behind the body. Due to the symmetry of the body, the boundary layer is formed and separated almost uniformly. 


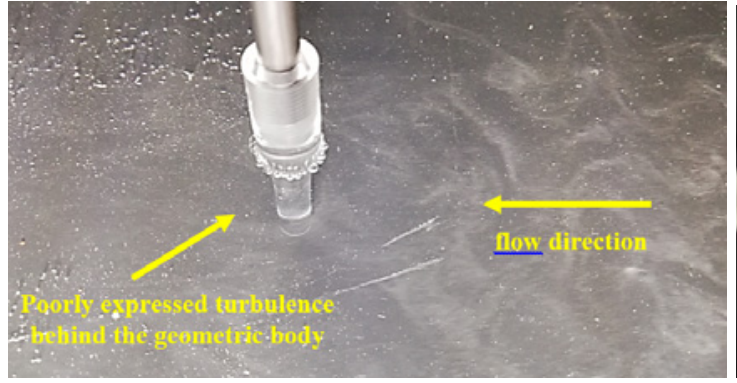

a)

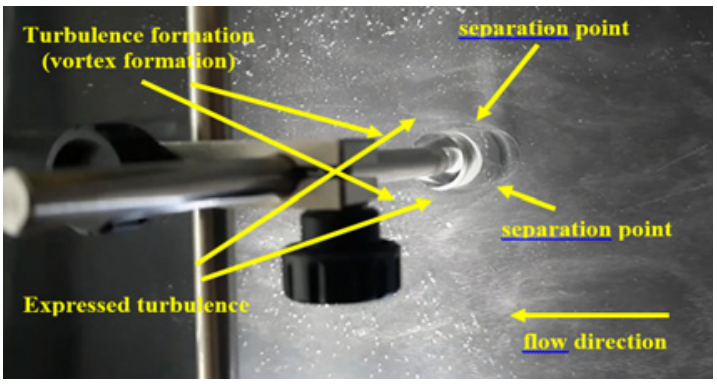

b)

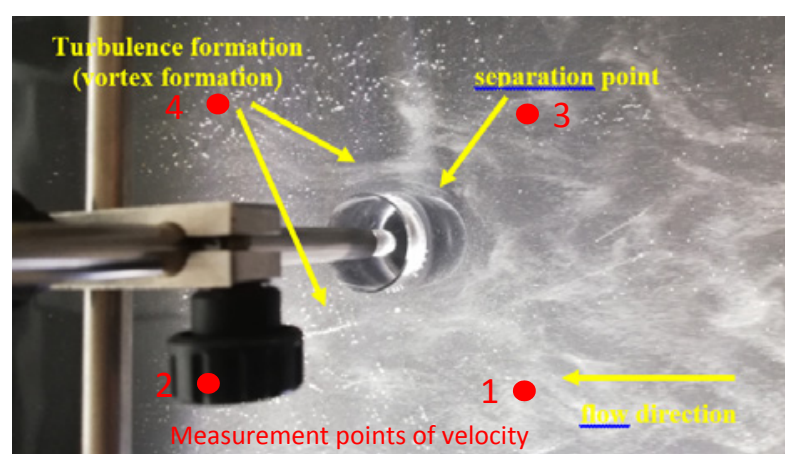

c)

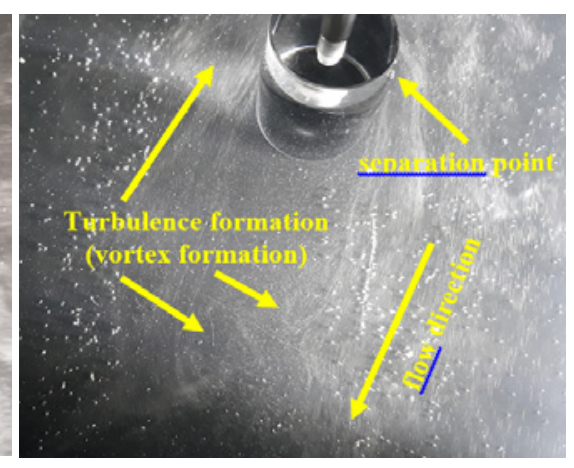

d)

Fig. 4 Fluid flow around cylindrical bodies, a) submodel $\left.2.1\left(d_{1}=6 \mathrm{~mm}\right), b\right)$ submodel $\left.2.2\left(d_{2}=12 \mathrm{~mm}\right), c\right)$ submodel $\left.2.3\left(d_{3}=18 \mathrm{~mm}\right), d\right)$ submodel $2.4\left(d_{4}=24 \mathrm{~mm}\right)$ [Foto: P. Černeka]

The second submodel (submodel 2.2) is a cylindrical body of $12 \mathrm{~mm}$ diameter placed at the center of the fluid flow (Figure 4b). Significant pressures occur in front of the body, while slight vortices appear behind the body. The figure shows that a boundary layer is formed equally on both sides of the body. The third submodel of the cylindrical body (submodel 2.3) of $18 \mathrm{~mm}$ diameter is also placed at the center of the fluid flow (Figure 4c). If the diameter of the analysed body grows the vortices are getting bigger behind the body. Separation of the boundary layer takes place in an area longer than a half of the diameter of the cylindrical body. The last tested submodel is a cylindrical body of $24 \mathrm{~mm}$ in diameter (submodel 2.4) located in the middle of the flow motion (Figure 4d). The characteristics that can be observed are similar to those of the previous submodels 2.2. and 2.3. The cylindrical bodies with a larger diameter are better for the observation of the vortex formed behind the body. Due to the symmetry of the body, the boundary layer is formed and separated almost uniformly.

\section{APPLICATION OF THE NUMERICAL MODEL}

Ansys Fluent is a software used for numerical simulations of fluid dynamics and is one of the tools contained within the ANSYS Workbench software package. Ansys Fluent uses a GreenGauss Finite Volume Method with a Cell-Centered formulation. In this paper, the Ansys Fluent 18.2 software is used to analyse fluid flow around 3D geometric bodies (Ansys CFX 15.0 Tutorials, 2013) [19]. Its advanced technology provides fast and accurate numerical simulations and is used to solve problems in the fields of hydromechanics, rheology, multiphase flow, flow propagation in open channels, fluid flow around solid and moving walls, aerodynamics, etc. Creating a numerical model in ANSYS Fluent involves three basic processes: 1. preprocessing, 2. solving a given problem, and 3. postprocessing. Preprocessing involves 
defining body geometry in a numerical model and generating a grid. The geometry of the body model is created in the DesignModeler and SpaceClaim programs. The options and attributes offered for drawing geometry are similar to those in the AutoCAD graphics program. Network generation is performed directly in the DesignModeler program (Figure 5). The problem is discretized by nodes that are connected in the form of finite elements that together form the volume of the domain. Each surface is assigned a name based on which the initial and boundary conditions on the model are later defined.
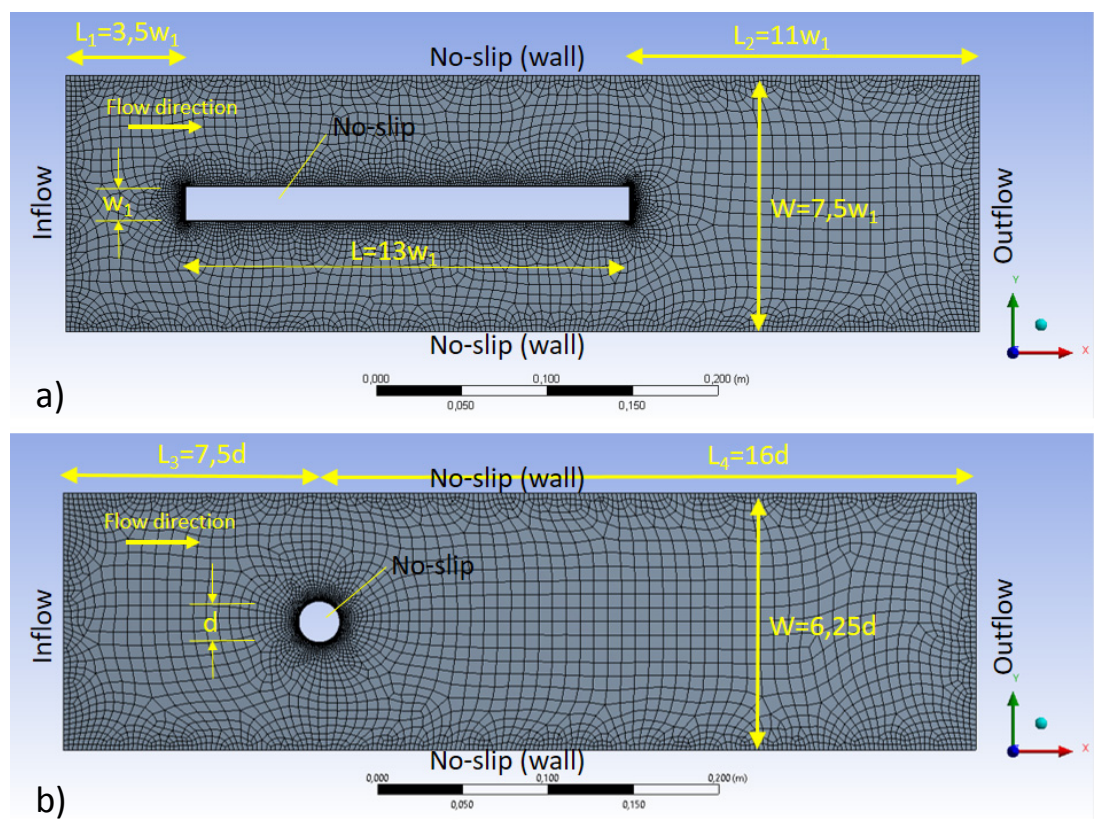

Fig. 5 Simulation domain and boundary conditions with network generation in the DesignModeler program, a) for Model1, b) for Model2 $(d=24 \mathrm{~mm})$

The total number of nodes and elements based on which the numerical network is discretized in the model of an elongated body is 10808 and 10176. In the model of cylindrical bodies, the number of nodes and elements varies depending on the diameter of the cylindrical body. At the smallest diameter $(d=6 \mathrm{~mm})$ the number of nodes is 5656 , i.e. the number of elements is equal to 5326 . In the model with the largest cylindrical body $(d=24 \mathrm{~mm})$, the number of nodes is 5276 , while the number of elements is 4945 . Meshing is one of the most important steps in obtaining an accurate boundary layer solution. For the generation of the flow domain, the triangular and quadrilateral finite elements are used.

For geometric bodies, programmatically controlled inflection is given, i.e. it is defined that in the area around the geometric bodies the numerical network has a different shape and is denser due to more precise results in the area of the bodies themselves. After generating the numerical grid, its quality is checked based on the asymmetry check. The asymmetry check represents the distance between the center of the face and the center of connection of two adjacent elements. If these two centers are at the same point, the asymmetry is 1,0 which is the worst possible for a numerical network. If the asymmetry is smaller, then the distance between the centers is greater and the quality of the numerical network is higher. The asymmetry value for the numerical network is approximately 0,26. In such a control, it is important to check the maximum asymmetry, because a large number of elements with high asymmetry can make it impossible to obtain accurate results. In order to improve the accuracy 
of the simulation results, it is necessary to thicken the network of elements around the observed geometric bodies.

The central part for creating a numerical model is defining the characteristics of a numerical model such as the type of turbulent model, initial and boundary conditions, fluid type, iterative method, and defining the accuracy of the convergence of the solution. Solving a given problem involves solving partial differential equations and finding a solution to a numerical model.

A steady-state flow regime is chosen for the type of hydrodynamic analysis. The numerical models in the paper are based on a density-based solver. In this paper, a $k-\varepsilon$ turbulent model with two characteristic equations is used to solve the turbulence problem using a standard wall function. The notation $k$ represents turbulent kinetic energy that gives information about how much energy is contained in the fluctuation. For the higher value of fluctuation intensity, the kinetic energy value increases. The coefficient $\varepsilon$ denotes the turbulent dissipation of kinetic energy, i.e. it represents the rate at which dissipation of kinetic energy takes place. The model presents two equations for $k$ and $\varepsilon$ :

$$
\frac{d k}{d t}=\frac{\partial k}{\partial t}+\overline{u_{j}} \frac{\partial k}{\partial x_{j}}=-\frac{\partial}{\partial x_{j}}\left(\frac{v_{t}}{\sigma_{k}} \frac{\partial k}{\partial x_{j}}\right)+P-\varepsilon
$$

where $\frac{\partial}{\partial x_{j}}\left(\frac{v_{t}}{\sigma_{k}} \frac{\partial k}{\partial x_{j}}\right)$ is diffusive transport, $P$ denotes the rate of production and $\varepsilon$ the rate of dissipation of kinetic energy. The left side represents a total change in turbulent kinetic energy. The second part is related to the change of $\varepsilon$ at time $t$ which can be written as:

$$
\frac{d \varepsilon}{d t}=\frac{\partial \varepsilon}{\partial t}+\overline{u_{j}} \frac{\partial \varepsilon}{\partial x_{j}}=-\frac{\partial}{\partial x_{j}}\left(\frac{v_{t}}{\sigma_{\varepsilon}} \frac{\partial \varepsilon}{\partial x_{j}}\right)+C_{\varepsilon 1} \frac{p \varepsilon}{k}-C_{\varepsilon 2} \frac{\varepsilon^{2}}{k}
$$

where $\frac{\partial}{\partial x_{j}}\left(\frac{v_{t}}{\sigma_{\varepsilon}} \frac{\partial \varepsilon}{\partial x_{j}}\right)$ is diffusive transport, $C_{\varepsilon 1} \frac{p \varepsilon}{k}$ represents the speed of production, while $C_{\varepsilon 2} \frac{\varepsilon^{2}}{k}$ is the rate of disappearance.

The $k$ - $\varepsilon$ turbulent model is very widely used, as they offer a good compromise between numerical effort and computational accuracy. The velocity and length scale are solved using separate transport equations. The $k-\varepsilon$ turbulent model in this research uses the gradient diffusion hypothesis to relate the Reynolds stresses to the mean velocity gradients and the turbulent viscosity. The turbulent viscosity is modelled as the product of turbulent velocity and turbulent length scale. The turbulence velocity scale is computed from the turbulent kinetic energy obtained from the solution of its transport equation. The turbulent length scale is estimated from two properties of the turbulence field, usually the turbulent kinetic energy and its dissipation rate. The dissipation rate of the turbulent kinetic energy is obtained from the solution of its transport equation.

In this paper, the fluid obstruction of the body is carried out in the presence of water and the body material is plastic (plexiglass). The most common initial condition is the initial velocity $[\mathrm{m} / \mathrm{s}]$, the wall roughness of the body, or turbulent intensity [\%]. To determine the turbulence on the model, a turbulence intensity of $5 \%$ is chosen, while the turbulent viscosity ratio is taken from the value of 10 . The following is the choice of the method for solving the partial differential equations of a numerical model. Depending on the simplicity or complexity of the 
generated numerical network for a given problem, the number of iterations, and the defined convergence accuracy, the calculation of the numerical model solution can take from a few seconds to a few hours.

As initial conditions on the model, the flow velocities of $0,25 \mathrm{~m} / \mathrm{s}$ (Model 1) and $0,5 \mathrm{~m} / \mathrm{s}$ (Model 2) are defined respectively. The outlet boundary of the channel is defined to be the pressure outlet, with an average reference (gauge) pressure of $0 \mathrm{~Pa}$. The left and the right-hand side walls of the flume and the walls of the geometric bodies have a no-slip boundary condition, where the flow velocity is zero at the wall surfaces and increases to the free-stream velocity away from the boundaries. The mesh cells used in numerical models are refined in order to resolve the boundary layer separation and the wake behind the oblong straight body and cylinders in the form of the vortex street.

For spatial discretization schemes, the First-Order Upwind Scheme and the Second-Order Upwind scheme are used. Based on these schemes the turbulent kinetic energy, turbulent dissipation rate, gradient, pressure, and Reynolds stresses are calculated. The results obtained using First-Order Upwind and Second-Order Upwind scheme are similar, so the First-Order Upwind scheme is chosen as a less time-consuming. This scheme is based on the principle that the face quantities are identical to the cell quantities, meaning that the cell-center values of any field variable represent a cell-average value and hold throughout the entire cell.

To solve the Navier-Stokes equations in numerical models, the SIMPLE algorithm (the SemiImplicit Method for Pressure Linked Equations) is used in this paper. The convergence tolerance in the convergence conditions for particular variables (continuity, $x$-velocity, $y$ velocity, $k$, and $\varepsilon$ ) are taken with residual values of 0,0001 . The number of iterations is taken from 1000 .

Standard initialization is taken as the solution initialization because it asks user to enter each value to define an appropriate environment and to augment the reality at a greater degree. In other words, if there is a problem in a controlled environment it is better to use the standard initialization (in relation to hybrid initialization) because the user can enter each value which might help provide a better specification to define the environment inside the numerical model.

The postprocessing involves the analysis and processing of data of individual physical quantities that can be displayed in vector and contour view or with simulations over streamlines for any selected transverse or longitudinal profile.

\subsection{HYDRODYNAMIC ANALYSIS ON PERFORMED NUMERICAL MODELS}

In order to compare the individual physical quantities on the submodels made on the physical model HM133, the paper additionally elaborated numerical models in the Ansys Fluent 18.2 software. The results of the hydrodynamic analysis are presented graphically through contour and vector displays (Figure 6 to 11).

\subsubsection{MODEL 1: OBLONG STRAIGHT BODY}

For the numerical 2D model of fluid flow around an elongated straight body, a $k-\varepsilon$ turbulent model with a standard wall function is chosen. The flow velocity at which the analysis is performed is $0,25 \mathrm{~m} / \mathrm{s}$ (measured velocity on the physical model HM133). Four submodels of the oblong straight body are analysed: submodel 1.1 (in the flow direction, Figure 6a), 
submodel 1.2. (at an angle of $10^{\circ}$ with respect to the direction of flow, Figure $6 \mathrm{~b}$ ), submodel 1.3. (at an angle of $20^{\circ}$ with respect to the direction of flow, Figure $6 \mathrm{c}$ ), and submodel 1.4. (at an angle of $25^{\circ}$ with respect to the direction of flow, Figure $6 \mathrm{~d}$ ). The most significant change in eddy viscosity can be observed on the oblong flat body set in the flow direction (Figure 6a). The images show a boundary layer formed. Moving the body for $10^{\circ}$ relative to the flow direction (Figure 6b) results in an earlier separation of the boundary layer. Ultimately, the analysis of the other submodels is similar. Moving the submodel by $10^{\circ}$, the development of larger vortices that occurs along with the separation point can be monitored more clearly. The change in flow velocity is conditioned by the position of the observed body (Figure 7).

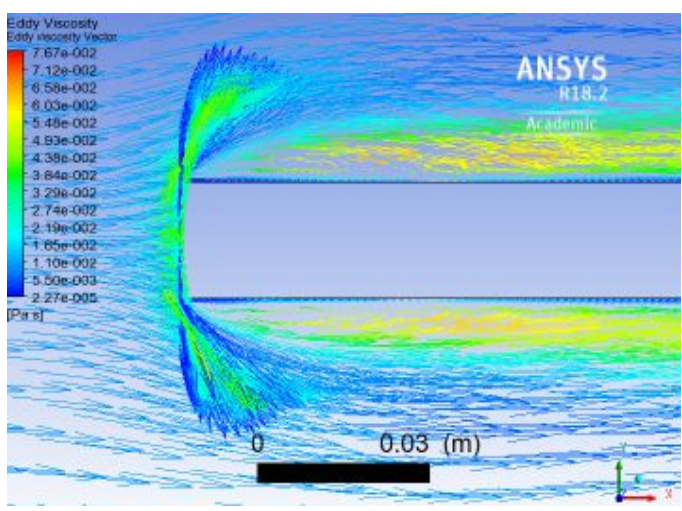

a)

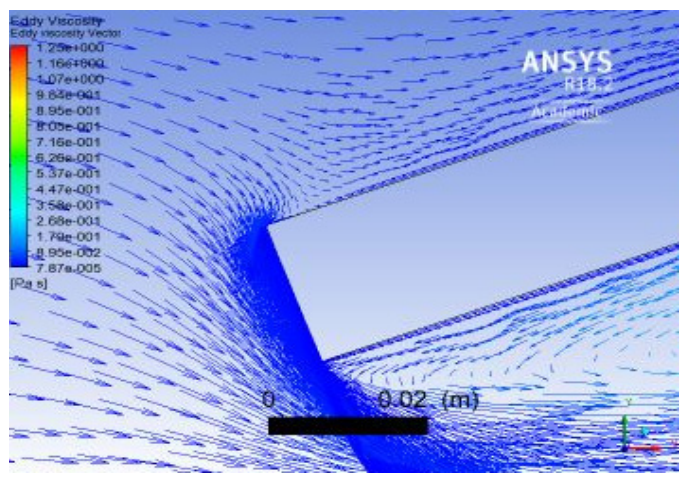

c)

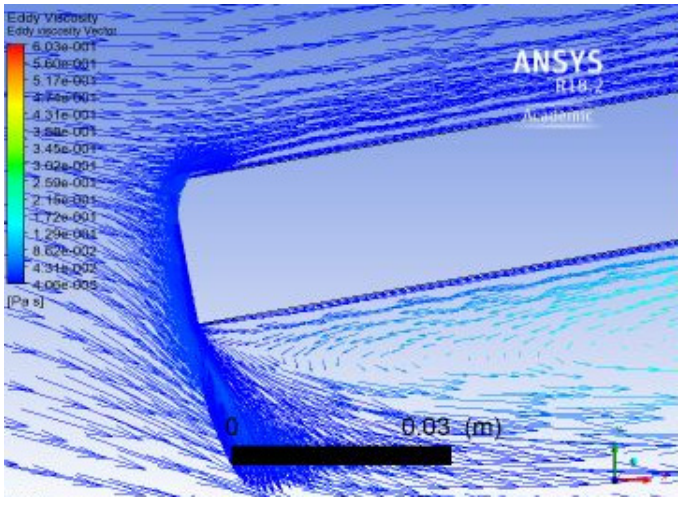

b)

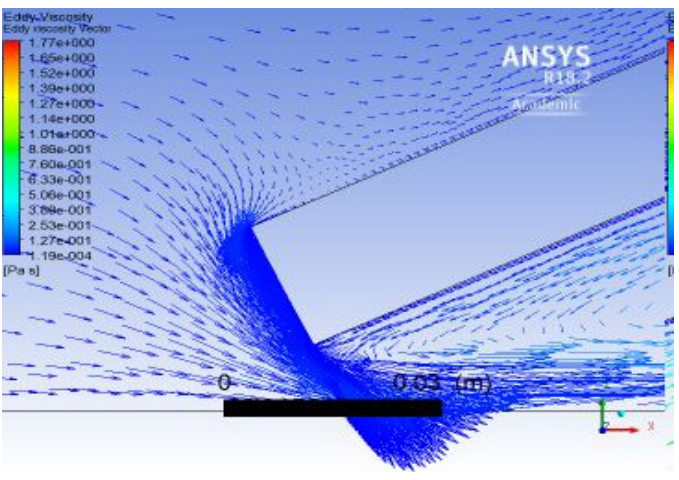

d)

Fig. 6 Vector view of the eddy viscosity change on the 1st numerical model

a) submodel 1.1, b) submodel 1.2, c) submodel 1.3 , d) submodel 1.4

On an elongated straight body set in the flow direction (Figure 7a)), the highest turbulence is created symmetrically at the peaks, resulting in a symmetric change in flow velocity which is also the highest at the peaks. If the geometric body is closer to the ends of the flow domain, the velocity vectors are more densely positioned and the velocity increases logically at these locations. Increasing the deflection of angle results in a decrease in the flow velocity acting on the upper body wall. An almost slight increase of the angle results in a large change in pressure on the part of the oblong straight body that is in direct contact with the fluid in motion (Figure 8). As can be assumed, the least pressure appears on submodel 1.1. (Figure 8a)) and the largest in submodel 1.4. (Figure 8d)). While there is a significant increase in pressure on one side of the body, on the other, from which vortices are formed, there is a decrease in pressure with an increase in angle (a feature of the Bernoulli equation). 

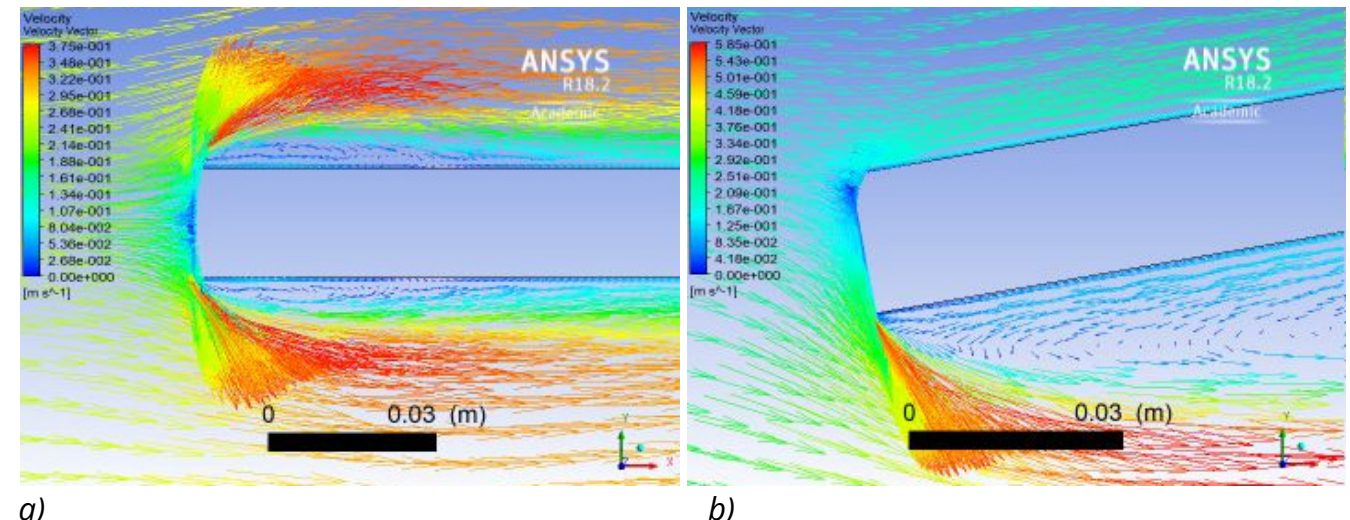

a)

b)

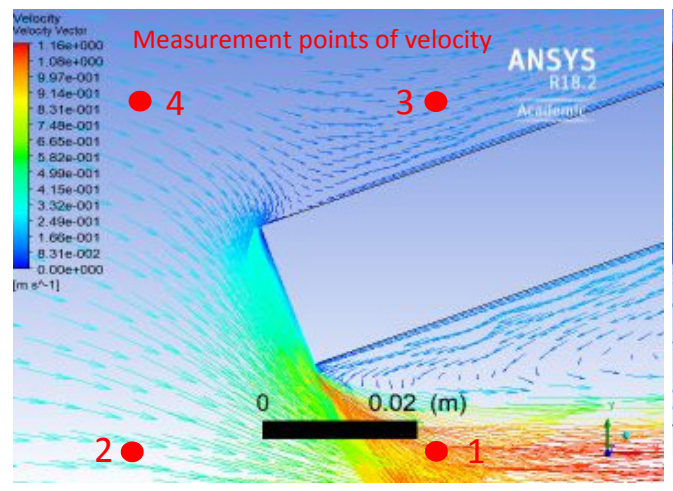

c)

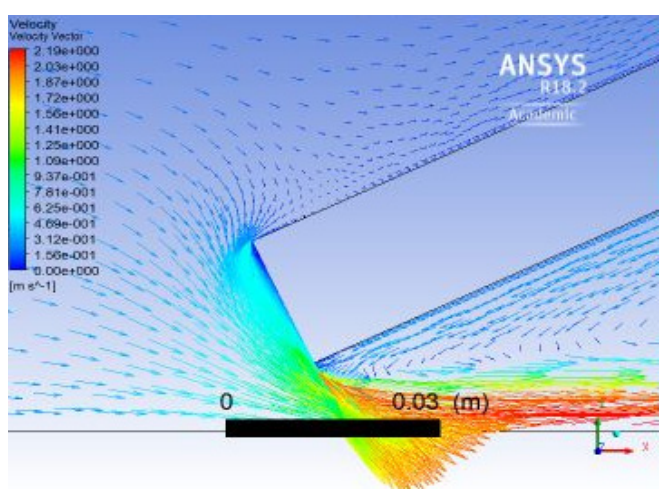

d)

Fig. 7 Vector view of the flow velocity change on the 1st numerical model a) submodel 1.1, b) submodel 1.2, c) submodel 1.3, d) submodel 1.4
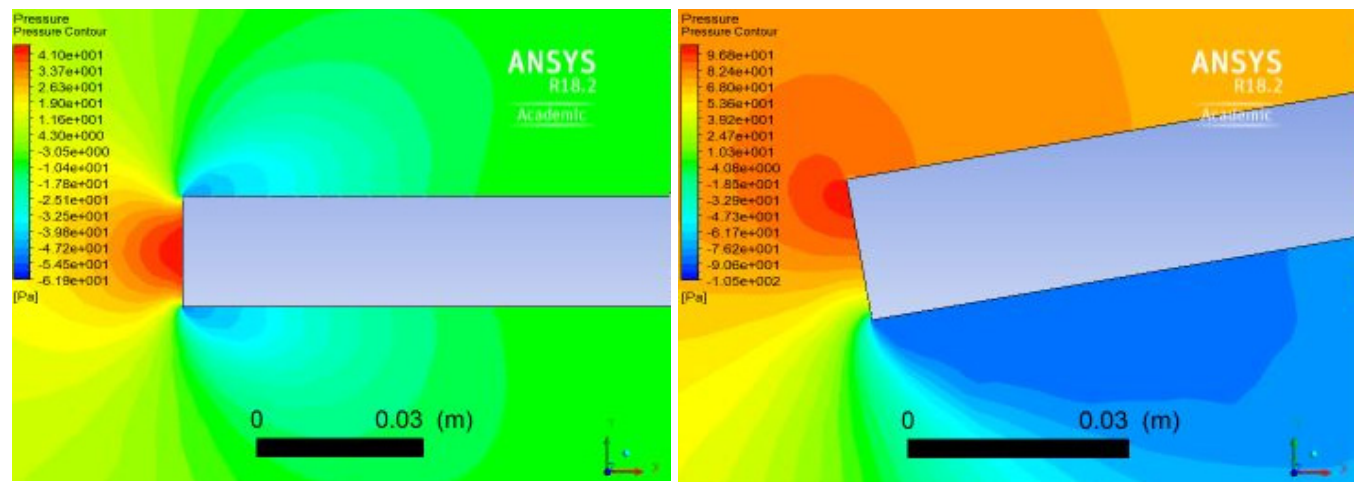

a)

b)
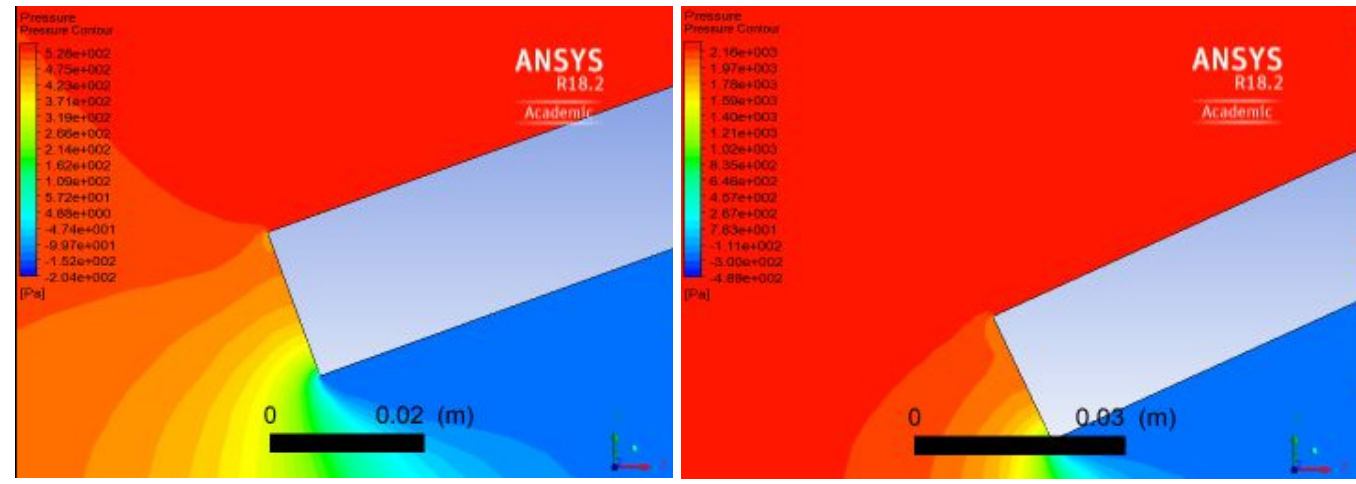

c)

d)

Fig. 8 Contour view of pressure change on the 1st numerical model a) submodel 1.1, b) submodel 1.2, c) submodel 1.3, d) submodel 1.4 


\subsubsection{MODEL 2: CYLINDRICAL BODIES WITH DIFFERENT DIAMETERS}

When designing a numerical 2D model of cylindrical bodies, a $k-\varepsilon$ turbulent model with a standard wall function is selected. The flow velocity at which the test is performed is $0,5 \mathrm{~m} / \mathrm{s}$ (velocity measured on the physical model HM133). Four submodels of cylindrical bodies are analysed: submodel 2.1 (with diameter $d_{1}=6 \mathrm{~mm}$, Figure 9a), submodel $2.2\left(d_{2}=12 \mathrm{~mm}\right.$, Figure 9b), submodel $2.3\left(d_{3}=18 \mathrm{~mm}\right.$, Figure 9c) and submodel $2.4\left(d_{4}=24 \mathrm{~mm}\right.$, Figure 9d). Figure 9 shows that if the diameters of the cylindrical bodies increases, the eddy viscosity increases, which is also observed during testing on the physical model HM 133. Eddy viscosity appears symmetrically on both sides of the cylindrical body due to its symmetrical position in the flow domain. The highest kinetic energy occurs behind the cylindrical bodies (Figure 9a-d). Almost similar characteristics can be observed on the vector view of flow velocity on the cylindrical bodies (Figure 10). On the cylindrical body with a diameter of $d_{1}=6 \mathrm{~mm}$ (Figure 10a) no significant vortex development is observed. As the diameter of the body increases, the development of vortices appearing behind the cylindrical bodies can be observed (Figure 10bd). In submodel 2.1. (Figure 10a) it can be observed that after a certain distance from the geometric body the fluid flow is normalized.
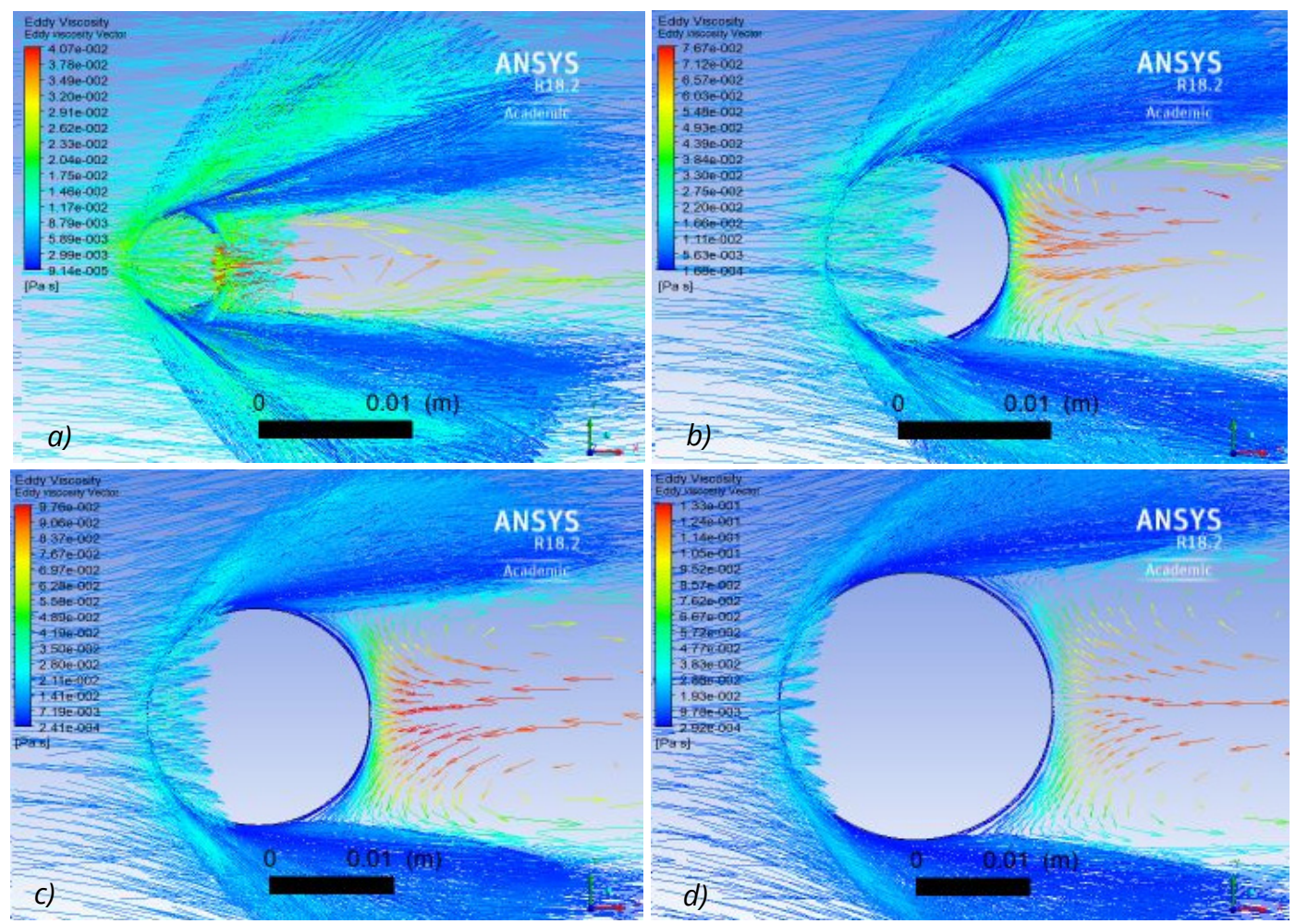

Fig. 9 Vector view of the eddy viscosity change on the 2nd numerical model

a) submodel 2.1, b) submodel 2.2, c) submodel 2.3, d) submodel 2.4 

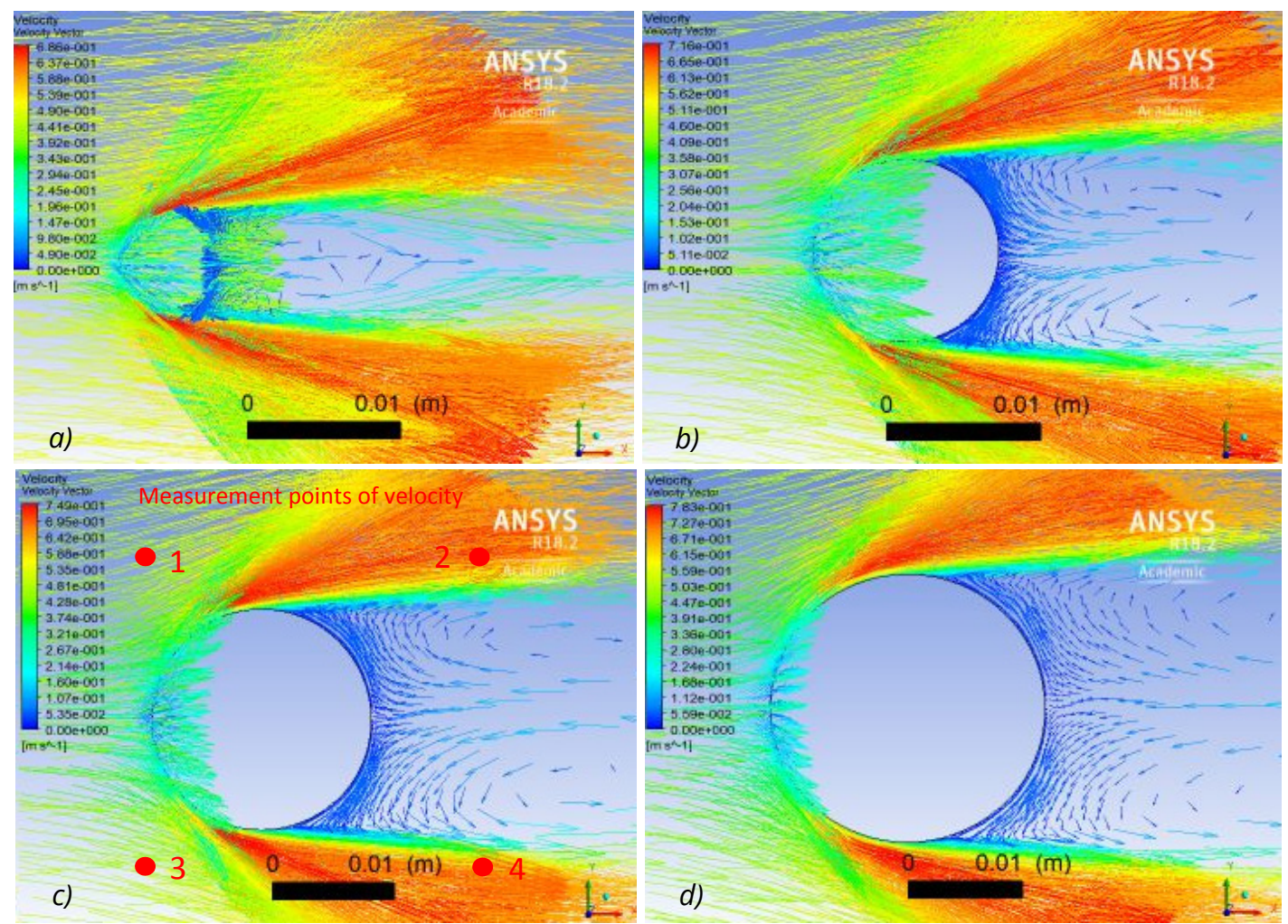

Fig. 10 Vector view of the flow velocity change on the 2nd numerical model

a) submodel 2.1, b) submodel 2.2, c) submodel 2.3, d) submodel 2.4

The contour views of the pressure change on a single cylindrical body of different diameters are shown in Figure 11. From Figures 11a) to d), it can be clearly seen that the symmetric pressure changes on both sides of the cylindrical bodies on all observed submodels. The highest pressure occurs at the contact of the cylindrical body being in direct contact with the incoming fluid streamlines. The lowest pressure occurs behind the cylindrical body where strong turbulence occurs and the flow velocity increases.

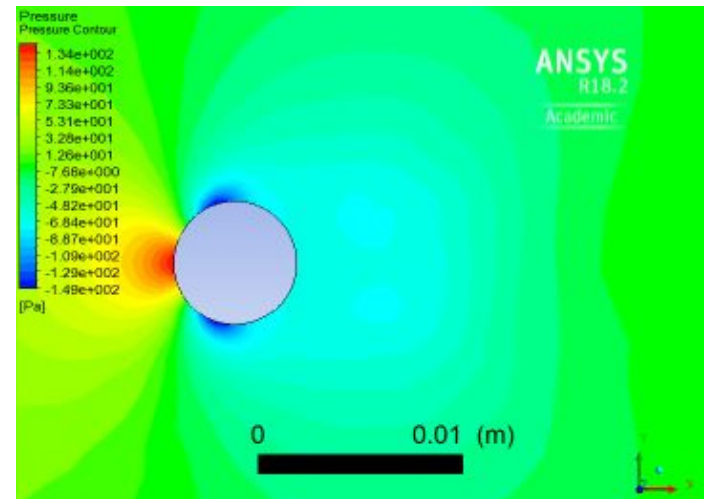

a)

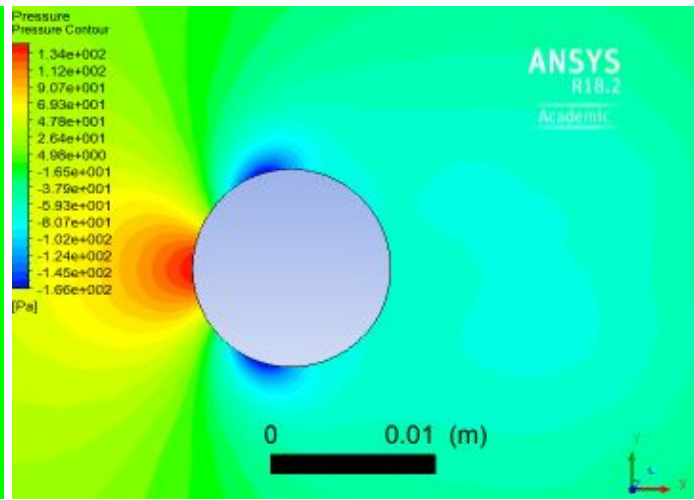

b) 


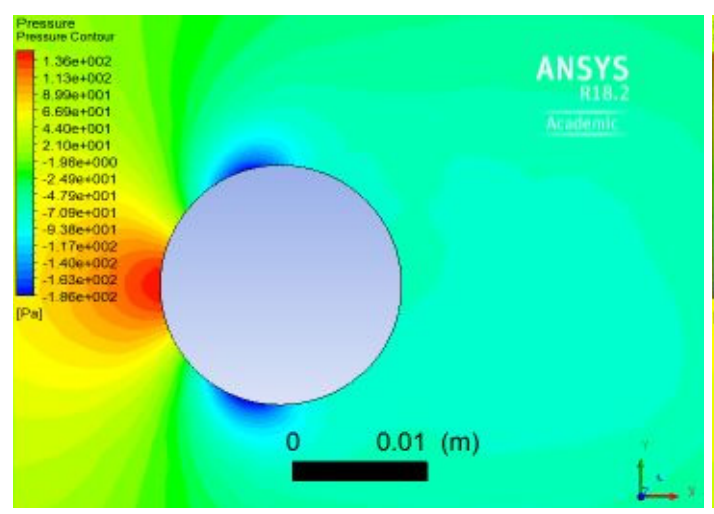

c)

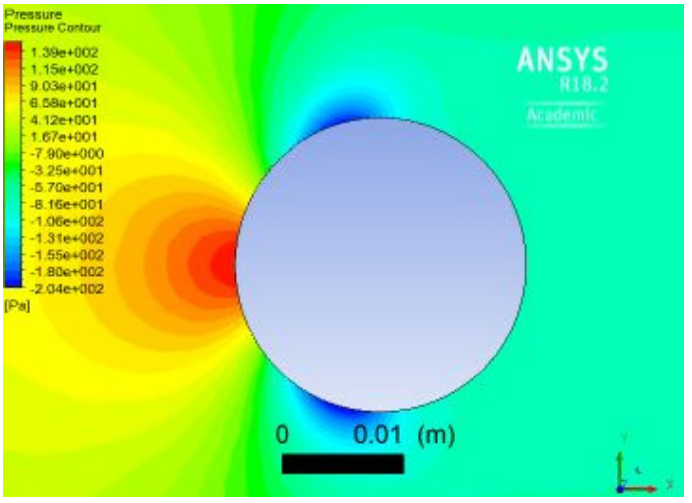

d)

Fig. 11 Contour view of pressure change on the 2nd numerical model

a) submodel 2.1, b) submodel 2.2, c) submodel 2.3, d) submodel 2.4

\section{CONCLUSION}

Hydrodynamic analyses of fluid flow were performed on the laboratory physical model HM 133 on two different geometric bodies: an elongated straight body and cylindrical bodies with different diameters. Several submodels were also made for each of the models, based on the angle of inclination of the individual geometric body with respect to the direction of water flow. Based on inlet velocity of $0,25 \mathrm{~m} / \mathrm{s}$, the width of the elongated body with $0,02 \mathrm{~m}$ and approximately coefficient of kinematic viscosity with $v=0,9^{*} 10^{-6} \mathrm{~m}^{2} / \mathrm{s}$ (corresponding to a room temperature of $25^{\circ} \mathrm{C}$ ) we could determine the value of Reynolds number for a given model of the elongated body with $R e=5500$. For models with cylindrical bodies (with diameters from 6 to $24 \mathrm{~mm}$ ) and an incoming velocity of $0,5 \mathrm{~m} / \mathrm{s}$, with the same upper value of the kinematic viscosity coefficient, the Reynolds numbers have the values in the range from 3300 to 13500 . The values of the Reynolds numbers calculated above indicate a turbulent flow behind the objects themselves. As the Re increases, flow begins to separate behind the cylinder and causes vortex shedding to take place. For the values $40<R e<200$ a laminar vortex shedding occurs in the wake of the cylinder. The laminar wake transient to turbulence takes place in the range of $200<R e<300$. The wake flow behind the cylindrical body grows into totally turbulent and a laminar boundary layer separation arises in the subcritical region $(R e>$ 300).

The main focus of the paper was to observe the characteristics of fluid flow around an individual body concerning the changes in its position. The formation of the boundary layer and the development of the separation point at which the boundary layer separated were also examined. If we look at the figures obtained by testing on a physical model and graphical contour views from numerical models, it can be concluded that the vortex separation points occur at very similar positions in the geometric body. Also, the vortex fields that occur at certain deflection angles concerning the flow velocity match very well in both physical and numerical models. In the model of elongated flat bodies, as the deflection angle increases with the flow direction, the total pressures on the lower part decrease due to a simultaneous increase in velocity (the part of channel contraction), i.e. on the opposite (upper) side of the body the pressures increase significantly. For larger deflection angles, significant vortex fields form along the wall of the lower part of the geometric body (greater length and width of the 
vortex zone), and the separation point is more distinct. In the case of circular cylindrical bodies, the contact pressure with water is more distinct at larger diameters of the cylinder, but the negative pressures caused by turbulence behind the separation point are also more significant compared to smaller diameters of the cylinder. With larger diameters of the cylindrical body, a larger length is required behind the body itself to re-establish the flow regime that prevailed before the water flowed around the body.

Numerical models have provided a more accurate solution than laboratory research. The smaller deviations of the individual physical quantities obtained from the results by numerical simulation and laboratory experiments are mainly attributed to the size of the numerical network. It should also be emphasized that in the laboratory examination of water flow around individual geometric bodies (primarily at small depths in the channel) special care should be taken not to create small waves within the physical model itself, as this may disrupt the hydrodynamic analysis itself. Therefore, small flow stabilization plates are placed at the beginning of the physical model. Because the walls of the simulation domain have no-slip conditions, the velocity of the flow is zero relative to the solid boundaries at the fluid-solid interface. Contour view of the velocity shows that the influence of the viscous forces between the fluid particles and the solid sidewalls of the open channel has negligible or no effect on the flow around the cylindrical bodies. The velocity gradually increases away from the channel walls.

In addition to the existing geometric bodies that come bundled with the physical model HM 133, it is possible to use the 3D printer within the Hydrotechnical Laboratory of the Faculty of Civil Engineering Rijeka to form various forms of interest geometric body for further study and testing. Accordingly, a combination (set) of different geometric bodies can be investigated and their legality can be seen in the fluids flow around them. Also, changes in fluid flow can be studied for the same body model and position by gradually increasing the flow velocity regulated by the controller.

\section{ACKNOWLEDGMENTS}

This paper is the result of a project on the Development of Research Infrastructure at the University Campus in Rijeka (RC.2.2.06-0001), co-funded by the European Regional Development Fund (ERDF) and the Ministry of Science and Education of the Rep. of Croatia.

\section{REFERENCES}

[1] E. Žic, $3^{\text {rd }}$ Summer School of Computational Fluid Dynamics, CFD Materials from lectures, Faculty of Technical Sciences in Novi Sad, Novi Sad, 5-10 July 2019.

[2] A.M. Bimbato, L.A.A. Pereira, M.H. Hirata, Study of the Vortex Shedding Flow Around a Body Near a Moving Ground, J. of Wind Engineering and Industrial Aerodynamics, Vol. 99, No. 1, pp. 7-17, 2011. https://doi.org/10.1016/i.jweia.2010.10.003

[3] P. Ausoni, Turbulent Vortex Shedding from a Blunt Trailing Edge Hydrofoil, PhD. Thesis, Ecole Polytechnique Federale de Lausanne, 189 pages, 2009.

[4] E. Błazik-Borowa, J. Bęc, T. Nowicki, T. Lipecki, J. Szulej, P. Matys, Measurements of flow parameters for 2-D flow around rectangular prisms of square and rectangle cross- 
sections located on the ground, Archives of Civil and Mechanical Engineering, Vol. 11, No. 3, pp. 533-551, 2011. https://doi.org/10.1016/S1644-9665(12)60100-9

[5] M.M. Zdravkovich, Flow Around Circular Cylinders: Fundamentals, Volume 2, Oxford University Press, New York, 2003.

[6] B.W.V. Oudheusden, F. Scarano, N.P.V. Hinsberg, D.W. Watt, Phase-Resolved Characterization of Vortex Shedding in the Near Wake of a Square Section Cylinder at Incidence, Experiments in Fluids, Vol. 39, pp. 86-98, 2005.

https://doi.org/10.1007/s00348-005-0985-5

[7] A.C. Benim, M. Cagan, A. Nahavandi, E. Pasqualotto, RANS Predictions of Turbulent Flow Past a Circular Cylinder Over the Critical Regime, Proc. of $5^{\text {th }}$ IASME/WSEAS, International Conference on Fluid Mechanics and Aerodynamics, Athens Greece, 2007.

[8] B.N. Rajani, A. Kandasamy, S. Majumdar, Numerical Simulation of Laminar Flow Past a Circular Cylinder, Applied Mathematics Modelling, Vol. 33, No. 3, pp. 1228-1247, 2009.

https://doi.org/10.1016/j.apm.2008.01.017

[9] M.C. Ong, T. Utnes, L.E. Holmedal, D. Myrhaug, B. Pettersen, Numerical Simulation of Flow Around a Smooth Circular Cylinder at Very High Reynolds Numbers, Marine Structures, Vol. 22, No. 2, 142-153, 2009.

https://doi.org/10.1016/i.marstruc.2008.09.001

[10] B. Gera, P.K. Sharma, R.K. Singh, CFD Analysis of 2D Unsteady Flow Around a Square Cylinder, International Journal of Applied Engineering Research, Vol. 1, No. 3, pp. 602610, 2010.

[11] H. Bai, J.W. Li, Numerical Simulation of Flow Over a Circular Cylinder at Low Reynolds Number, Advanced Material Research, Vols. 255-260, pp. 942-946, 2011.

https://doi.org/10.4028/www.scientific.net/AMR.255-260.942

[12] I.M. Kozlov, K.V. Dobergo, N. Gnesdilov, Application of RES Methods for Computation of Hydrodynamic Flows by an Example of 2D Flow Past a Circular Cylinder for Re=5-200, International Journal of Heat and Mass Transfer, Vol. 54, No. 4, pp. 887-893, 2011.

https://doi.org/10.1016/j.ijheatmasstransfer.2010.10.013

[13] A.A. Mustto, G.C.R. Bodstien, Subgrid Scale Modeling of Turbulent Flow Around Circular Cylinder by Mesh-Free Vortex Method, Engineering Applications of Computational Fluid Mechanics, Vol. 5, No. 2, pp. 259-275, 2011.

https://doi.org/10.1080/19942060.2011.11015369

[14] S. Wornom, H. Ouvrard, M.V. Salvetti, B. Koobus, A. Dervieux, Variational Multiscale Large-Eddy Simulations of the Flow Past a Circular Cylinder: Reynolds Number Effects, Computers and Fluids, Vol. 47, No. 1, pp. 44-50, 2011.

https://doi.org/10.1016/j.compfluid.2011.02.011

[15] M.I. Yuce, D.A. Kareem, A Numerical Analysis of Fluid Flow Around Circular and Square Cylinders, American Water Works Association AWWA, pp. 546-554, 2016.

https://doi.org/10.5942/jawwa.2016.108.0141

[16] M. Bukurov, E. Žic, Fluid mechanics - theoretical foundations, Working Manual, Faculty of Civil Engineering, University of Rijeka, Rijeka, 248 pages, 2017. 
[17] P. Černeka, Hydrodynamic analysis of fluid flow around different geometric bodies, Final thesis, Faculty of Civil Engineering, University of Rijeka, Rijeka, 88 pages, 2019.

[18] M. Pamić, E. Žic, I. Biluš, L. Lešnik, Physical model of forming the boundary layer, Proceedings of the Faculty of Civil Engineering, University of Rijeka, Rijeka, Vol. 20, No. 1, pp. 43-58, 2018. https://doi.org/10.32762/zr.20.1.3

[19] Ansys CFX 15.0 Tutorials 2013, Ansys Inc. Southpointe Canonsburg 2013. 